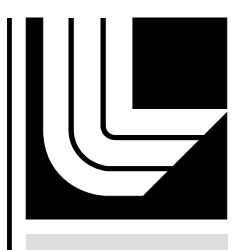

LAWRENCE LIVERMORE N A TIO N A L LABORATORY
Towards SiC Surface

Functionalization: An Ab Initio Study

G. Cicero, A. Catellani

February 18, 2005

The Journal of Physical Chemistry 
This document was prepared as an account of work sponsored by an agency of the United States Government. Neither the United States Government nor the University of California nor any of their employees, makes any warranty, express or implied, or assumes any legal liability or responsibility for the accuracy, completeness, or usefulness of any information, apparatus, product, or process disclosed, or represents that its use would not infringe privately owned rights. Reference herein to any specific commercial product, process, or service by trade name, trademark, manufacturer, or otherwise, does not necessarily constitute or imply its endorsement, recommendation, or favoring by the United States Government or the University of California. The views and opinions of authors expressed herein do not necessarily state or reflect those of the United States Government or the University of California, and shall not be used for advertising or product endorsement purposes. 


\title{
Towards SiC surface functionalization: an ab initio study
}

\author{
Giancarlo Cicero \\ Lawrence Livermore National Laboratory, \\ P.O. Box 808, Livermore CA94550 USA \\ Alessandra Catellani* \\ CNR-IMEM, Parco Area delle Scienze, 37A, I-43010 Parma, Italy
}

(Dated: December 13, 2004)

\begin{abstract}
We present a microscopic model of the interaction and adsorption mechanism of simple organic molecules on $\mathrm{SiC}$ surfaces as obtained from ab initio molecular dynamics simulations. Our results open the way to functionalization of silicon carbide, a leading candidate material for bio-compatible devices.
\end{abstract}

PACS numbers: $68.43 . \mathrm{Bc}, 68.47 . \mathrm{Fg}$ 
The research on functionalization of semiconductor surfaces has been an extremely active field in the last decade [1] to devise novel hybrid devices for advanced technological applications, and in particular in biotechnology. Inspired by the realization of self-assembled monolayers on metal (gold) surfaces [2-4], most of the recent work on semiconductor surfaces has been related to thiol deposition: molecular adsorption of thiols in a bridge-like structure on top of silicon [5] and gallium arsenide surfaces $[6,7]$ has been described by means of stimulated adsorption/desorption, photoemission, atomic-force microscopy and multiple internal reflection. Furthermore, attention has been devoted also to ammine interaction with silicon [8] and gallium arsenide surfaces [9-11] in view of potential applications in chemical sensors and nitride growth. Still, little is known about the microscopic mechanism of the adsorption process, and about the structural and electronic details of the adsorbed species.

Silicon carbide is considered to be a very promising material for biophysics applications, due to its low weight, high strength, extreme hardness, wear and corrosion resistance, and inertness [12]. The SiC cubic polytype, in particular, presents a number of stable different reconstructions, offering carbon or silicon outermost layers with different stoichiometries, thus with many different electronic properties, when exposed to external ambient. Nevertheless, little is known regarding the reactivity of $\mathrm{SiC}$ surfaces with water and with possible organic molecules to be adopted in functionalization schemes. Only very recently experimental and theoretical studies have been able to characterize SiC surfaces exposed to water vapor and these investigations have concentrated on the cubic polytype (001) surfaces. Experiments on the Si-rich (3x2)-reconstructed $\mathrm{SiC}(001)$ surface [13] showed that dissociation of water molecules leads to perfect passivation of the outermost excess Si layer. These results are consistent with those of $a b$ initio simulations of water molecules on stoichiometric silicon terminated $\mathrm{SiC}(001)$ ( $\mathrm{Si}-\mathrm{SiC}(001)$ ) which revealed how the dissociation mechanism occurs, and the role played by the surface reconstruction and substrate polarity $[14,15]$.

Here, we report on first principles predictions of the chemical processes which eventually lead to adsorption of organic molecules on the $\mathrm{Si}-\mathrm{SiC}(001)$ surface, in the spirit to propose feasible ways to surface functionalization. We focussed our attention on simple molecules of the form $\mathrm{CH}_{3}-\mathrm{X}$, where $-\mathrm{X}$ were chosen to represent the most important functional groups found in bio-molecules, like aminoacids. In particular we considered acetic acid $\left(\mathrm{CH}_{3}-\mathrm{COOH}\right)$, methanol $\left(\mathrm{CH}_{3}-\mathrm{OH}\right)$, methanethiol $\left(\mathrm{CH}_{3}-\mathrm{SH}\right)$ and methylamine $\left(\mathrm{CH}_{3^{-}}\right.$ $\mathrm{NH}_{2}$ ). Our results indicate that all these functional groups interact attractively with the 
$\mathrm{Si}-\mathrm{SiC}$ surface and eventually give dissociation products: the polarity of the substrate has a peculiar role in activating adsorption and reaction.

\section{METHOD}

Our calculations have been performed within Density Functional Theory in the Generalized Gradient corrected Functional (PBE), as parameterized by Perdew et al. [16]. In our simulations, the $\mathrm{SiC}(001)$ surfaces were represented by a symmetric slab, periodically repeated along the (001) direction. We used supercells with 11 layers, 8 atoms/layer and a $\simeq 13.7 \AA$ vacuum region between the clean surfaces, required to avoid interaction between periodic replicas. We considered one single molecule impinging on each slab surface, at different distances from the Si-dimers, simulating 1/4 ML coverage (1 ML coverage is defined as one molecule adsorption per Si dimer). Similar to previous investigations $[14,15]$, the integration over the Brillouin Zone was performed using only the $\Gamma$ point, which corresponds to 3 inequivalent $\mathrm{k}$-points in the primitive cell. We used nonlocal pseudopotentials [17] derived within the PBE approximation. The electronic wavefunctions (charge density) were expanded in plane waves, with energy cutoff up to 80 (320) Ry.

The geometry of all configurations was optimized using ab initio molecular dynamics $[18,19]$ at the theoretical $\mathrm{SiC}$ equilibrium lattice parameter. All atoms were allowed to move, and geometries were considered at convergence when forces were less than $10^{-4} \mathrm{a} . \mathrm{u}$./atom.

Within this frame, we first optimized the structure of the $\mathrm{CH}_{3}-\mathrm{X}$ molecules in the gas phase (the optimized structures agreed within $2 \%$ with experimental gas phase data). We then simulated the interacting surface $/ \mathrm{CH}_{3}-\mathrm{X}$ systems eventually analyzing the dissociation products. This also allows us to evaluate binding energies, once the interaction (adsorption) with the substrate takes place: the binding energy is defined as

$$
E_{b}=\frac{1}{N_{m o l}}\left(E_{t o t}-E^{S i C}-N_{m o l} E^{m o l}\right),
$$

where $E_{t o t}$ is the total energy of the interacting (reacted) system, $E^{S i C}$ is the total energy of the clean $\mathrm{Si}-\mathrm{SiC}(001)$ surface, $E^{\text {mol }}$ is the total energy of the selected isolated molecule, and $N_{m o l}$ is the number of molecules "deposited" on the surfaces.

As for the substrate, we considered the $\mathrm{Si}-\mathrm{SiC}(001)$ surface in the $p(2 \times 1)$ reconstruction: the equilibrium structure presents surface unbuckled Si-dimers $2.64 \AA$ long, and a small 
band gap between antibonding surface states with $\pi_{x, y}^{*}$ and $\pi^{*}+\sigma$ character localized on dimers, with partial bonding charge along the dimer rows [15, 20].

The simulation of the interacting systems consisted of a molecular dynamics simulation at finite temperature, sampling selected local minima of the total energy surfaces: starting from the initial ground-state configuration of the two non interacting systems, with the molecule at $\simeq 3 \AA$ above the surface, we considered the most relevant adsorption sites, i.e. on top and between surface dimers, and let the system relax. But for the specific case of methylamine, described below, we considered the molecules impinging with the desired functional group facing the surface. These are the most reactive sites of a molecule: indeed, the electronic structure analysis of the isolated molecules shows that the highest occupied and lowest unoccupied molecular orbitals (HOMO and LUMO) are mainly localized on the -X part of the molecule, which consequently is expected to react with the $\mathrm{Si}-\mathrm{SiC}$ surface, once the molecule approaches it.

For a complete discussion of the method adopted and the tests performed, we remand to $[14,15]$.

\section{RESULTS}

To allow for a quick comparison and a better understanding of the interaction mechanism of bio-molecules on $\mathrm{Si}-\mathrm{SiC}$, we recall first the main results obtained for water adsorption.

\section{Water}

As we have recently shown $[14,15]$, water at any coverage spontaneously dissociates on the Si-SiC(001) surface, with a barrier-less energy gain of $\simeq 3.5 \mathrm{eV} /$ molecule $(80.7 \mathrm{Kcal} / \mathrm{mol})$. At low coverage, once dissociation takes place, the hydroxyl group preferentially binds to one Si-dimer, while the $\mathrm{H}$ radical saturates one of the adjacent Si-dimer dangling bonds. As the molecule approaches the surface, the surface silicon dimers become buckled. The induced buckling imparts a polarity to the dimers due to an electronic transfer occurring from the down to the up $\mathrm{Si}$ atom, which is responsible for the $\mathrm{Si}-\mathrm{Si}$ bond shortening and the attractive polar interaction with the adsorbed water molecule. Such electronic transfer is revealed by the presence of occupied electronic states close to the highest occupied molecular 
orbital (HOMO; see for example Fig. 1) where a heap of electronic charge on the up Si atom of the dimer is found. Once the dissociation occurs the -OH groups may interact through a hydrogen like bond with a neighboring silicon dimer, inducing a large distortion in the substrate reconstruction.

At full coverage, the adsorbates saturate all the surface dangling bonds, re-orienting themselves to form a fully periodic network of hydrogen bonds, with further modification of the structural and electronic surface properties. Due to the saturation of all the dangling bonds upon water adsorption, at full coverage the optical gap of the surface increases, giving rise to a wide band gap substrate (the gap computed with the approximations used in our calculation is $1.6 \mathrm{eV}$ ). The highest occupied electronic states are surface $\pi$ states strongly localized on Si dimers, which are shortened in this case. These states are hybridized with the oxygen p-orbital, giving rise to charge spilling around the - $\mathrm{OH}$ group (and in part at the Si-H fragment).

\section{Acetic Acid}

In our further simulations, the molecules are "deposited" on top and between the rows, to mimic water adsorption and dissociation. In close similarity to the results obtained for water at low coverage, when one (any) of the aforementioned molecules is impinging on the silicon terminated stoichiometric $\mathrm{SiC}(001)$ surface, we observe a strong modification of the surface dimers, which tend to tilt, in order to maximize the interaction with the $-\mathrm{X}$ functional group of the molecule.

When considering acetic acid deposition, we let the molecule approach the surface with the - $\mathrm{OH}$ bond nearly parallel to the surface dimers; at distances higher than $2.0 \AA$ the molecule/surface interaction has an electrostatic character: as for the water case, a dipoledipole interaction is established between the polar group and the induced dipole at the buckled surface silicon dimer. As the molecule is attracted by the surface, a dative bond between the -X lone pair and a silicon atom may be eventually formed, with a Si-O distance of about $1.9 \AA$.

Dative bond formation represents a metastable structure with an energy gain of about $1.1 \mathrm{eV} /$ molecule $(25.4 \mathrm{Kcal} / \mathrm{mol})$ : indeed, when the acetic molecule is adsorbed between rows, spontaneous dissociation is observed. The reaction which leads to adsorption and 
dissociation of the acetic acid is exothermic, with an energy gain of $2.7 \mathrm{eV} /$ molecule $(62.3$ $\mathrm{Kcal} / \mathrm{mol}$ ). Upon dissociation, the acid hydrogen of the -COOH group is released and bonds to a Si-dimer, the remaining radical bonded to an adjacent dimer (see left panel of Fig.2). In the final structure the $\mathrm{Si}-\mathrm{H}$ bond is $1.47 \AA$ long and the $\mathrm{Si}-\mathrm{O}$ distance is $1.68 \AA$, while the reacted silicon dimers become shorter $(\simeq 2.45 \AA)$. The $\mathrm{C}=\mathrm{O}$ bond-length and the remaining structural details of the dissociated molecule do not change: this is a common feature in all the dissociative adsorption processes here described.

\section{Methanol}

At variance with acetic acid, methanol does not dissociate spontaneously on $\mathrm{Si}-\mathrm{SiC}(001)$. Rather, an intermediate physisorbed configuration with the molecule bridging two Si atoms can be found (see Fig. 2, right panel). As for water and acetic acid, in other adsorption geometries the molecules may be "trapped" in an adsorption state, characterized by formation of a dative bond between the $\mathrm{Si}$ and $\mathrm{O}$ atom, with shortening and buckling of the involved Si-dimers. The interaction energy is $-0.8 \mathrm{eV} /$ molecule $(18.2 \mathrm{Kcal} / \mathrm{mol})$, which is comparable to the value obtained in similar configurations for acetic acid.

When methanol dissociation occurs (between Si-dimer rows, with cleavage of the $\mathrm{OH}$ group), the reaction, although activated, is strongly exothermic. The energy gain is $\mathrm{E}_{\text {gain }}=3.1 \mathrm{eV} /$ molecule $(71.7 \mathrm{Kcal} / \mathrm{mol})$, similar to the one we obtained for water chemisorption $[14,15]$ : the optimized geometry is characterized by formation of an O-Si bond $1.64 \AA$ long, and angles $\mathrm{C}-\mathrm{O}-\mathrm{Si}=139^{\circ}$, while $\mathrm{H}-\mathrm{Si}=1.48 \AA$; the substrate is modified, with induced alternate buckling of the dimers: the one which is bonded to $\mathrm{H}$ has the Si atom involved in the bond higher $(\delta \mathrm{z}=+0.08 \AA)$, while the following dimer along the row has the equivalent $\mathrm{Si}$ atom lower $(\delta \mathrm{z}=-0.15 \AA)$; the dimers are respectively 2.46 and $2.62 \AA$ long (central panel in Fig. 2). To evaluate the energy barrier for this reaction, we performed a constrained molecular dynamics simulation starting with the impinging molecule between dimer rows and increasing the $\mathrm{O}-\mathrm{H}$ distance $\left(\mathrm{d}_{O H}\right)$. At each step, all the atoms were allowed to relax, while $\mathrm{d}_{O H}$ was kept constant. In this way, we estimated an energy barrier of $\simeq 0.3$ $\mathrm{eV} / \mathrm{molecule}(6.9 \mathrm{Kcal} / \mathrm{mol})$ for dissociation. 


\section{Methanethiol}

For methanethiol on the contrary the interaction strength with $\mathrm{Si}-\mathrm{SiC}$ is surprisingly high even at large $(\geq 3 \AA)$ distances. This is in agreement with the general notion that thiols are more acid than the relative alcohol (in this case methanol previously discussed). We interpret the spontaneous dissociation of thiolate groups on $\mathrm{Si}-\mathrm{SiC}(001)$ as highly activated by the polar character of the surface: at variance with $\mathrm{Si}(001)$, here the silicon atoms tend to be electron-deficient, thus favoring bond formation with S. The energy gain upon complete $\mathrm{SH}$ dissociation is $2.8 \mathrm{eV} /$ molecule $(65.1 \mathrm{Kcal} / \mathrm{mol})$.

The chemisorption configuration (see Fig. 3, right panel) is characterized by a geometrical structure where S-Si is $2.15 \AA$ long and the angle CSSi is $\simeq 105^{\circ}$; the $\mathrm{Si}-\mathrm{H}$ bond length is $1.49 \AA$, similar to the other cases, and the Si dimers involved in the dissociation process are buckled and shortened, as for all the other systems described: in the present case, however, we can observe an overall lower substrate distortion with respect e.g to $\mathrm{H}_{2} \mathrm{O}$.

During the minimization procedure, we may observe the system "trapped" in a different metastable configuration (see Fig. 3-left) characterized by a "bridge" geometry with equivalent Si-S distances of $\simeq 2.4 \AA$ and almost unperturbed Si-dimers. Similar "bridge" geometries were also found as molecular adsorbed structures in previous work for methanethiol on silicon and GaAs [6] and as metastable solutions in a combined computational and experimental work for methylamine adsorption on $\mathrm{Si}(001)$ [8].

\section{Methylamine}

Finally, we studied methylamine. Strong modifications of the substrate occur when the molecules are approaching the surface: No spontaneous dissociation was observed, although $\mathrm{N}-\mathrm{Si}$ dative bond is readily formed, when the molecule is impinging between dimer rows. Molecule dissociation was tested in two possible geometries, according to [8]: both reactions are exothermic, with $E_{\text {gain }}$ of $3.3 \mathrm{eV} /$ molecule $(75.1 \mathrm{Kcal} / \mathrm{mol} \mathrm{CN}$ dissociation) and 2.7 $\mathrm{eV} /$ molecule $(62.3 \mathrm{Kcal} / \mathrm{mol}$ for $\mathrm{NH}$ dissociation) respectively. The behavior is quite similar to what observed for $\mathrm{Si}(001)$ in DFT-GGA [8], although with slightly higher energy gain for

both dissociation mechanisms. When dissociation occurs, an N-Si bond $1.73 \AA$ long is formed upon NH cleavage, along with strong substrate modifications. When dissociative adsorption 
is instead obtained via CN cleavage, the substrate is distorted to a lower extent, with smaller buckling and dimer shortening, in agreement with the fact that heavier fragments bond to two different dimers (see Fig. 5). The final C-Si distance is $1.87 \AA$, close to a $\mathrm{SiC}$ bond length, while N-Si is $1.73 \AA$, as for the NH cleavage.

In order to evaluate possible paths towards $\mathrm{CN}$ dissociation, we have performed further molecular dynamics simulations, with the impinging molecule which exposes the $\mathrm{CN}$ bond parallel to the surfaces, between rows, starting with $\mathrm{Si}-\mathrm{N} \simeq 2.1$ and $\mathrm{C}-\mathrm{Si} \simeq 2.4 \AA$ distances. In free molecular dynamics, the molecule tends to move away from the surface, increasing the C-Si distance, with both tilt of the molecule, and of the underlying Si-dimer, because of a repulsive effect between the hydrogen atoms in the $-\mathrm{CH}_{3}$ group and the slightly positive Si atoms in the substrate. We thus resorted to constrained molecular dynamics, imposing the cleavage of the C-N bond upon adsorption, starting from the "flat" molecular geometry described above. In this way, we may estimate an energy barrier towards $\mathrm{C}-\mathrm{N}$ cleavage of about $\simeq 1 \mathrm{eV} /$ molecule $(23.1 \mathrm{Kcal} / \mathrm{mol})$, as due to dissociation of the $\mathrm{CN}$ bond, and rotation of the $-\mathrm{CH}_{3}$ radical. Once dissociative adsorption is obtained, the structural details are close to the ones described above. For both free and constrained molecular dynamics, finally, the structural details of the fragments do not change with respect to the free molecule, as in all the other cases presented above.

With a similar procedure, we estimate the energy barrier for $\mathrm{NH}$ cleavage to be $\simeq 0.4$ $\mathrm{eV} / \mathrm{molecule}(9.2 \mathrm{Kcal} / \mathrm{mol})$.

Similar to what discussed by Mui et al. [8] for the attachment of methylamine on the $\mathrm{Si}(001)$ surface, our results show that although dissociation involving $\mathrm{C}-\mathrm{N}$ bond cleavage is thermodinamically more favorable than the N-H dissociation pathway, the activation barrier for the $\mathrm{C}-\mathrm{N}$ one is higher than that for $\mathrm{N}-\mathrm{H}$ dissociation. Consequently a selective cleavage of the N-H bond may be achieved by controlled experimental conditions.

\section{Electronic structure}

The electronic structure analysis of the reacted systems reveals common features, independently on the molecule used to achieve functionalization. Similarly to what was found for water molecules at low coverage adsorption, the gap of the system is increased from 0.3 $\mathrm{eV}$ to about $0.5 \mathrm{eV}$. This is related to the saturation of some surface dangling bonds when 
dissociation occurs, as also evidenced by the density of states analysis (not shown here). The DOS reveals a decrease of the intensity of the peak of the highest occupied states of the clean substrate, which in fact are surface states localized on the surface silicon dimers. The HOMO and the LUMO states of the reacted surface, at 1/4 ML coverage, are still surface states and are localized onto the unreacted silicon surface dimers. Those states do not show anymore the $\mathrm{p}(2 \times 1)$ surface periodicity: this feature may be detected by STM experiments.

The highest occupied molecular orbital of the adsorbing molecule, that was localized on the -X group before adsorption, appears to be still highly localized on the $\mathrm{O}(\mathrm{S}, \mathrm{N})$ atom as shown for example in Fig. 3 for the case of methanethiol. This state is an occupied state in the gap, being located above the top of the substrate valence band $(\sim 0.3 \mathrm{eV})$. The position of this state appears to be almost independent from the molecule. The opening of the gap, along with the presence of these surface states and associated changes in work function should be detectable in photoemission experiments, as markers of the reaction.

\section{STABILITY OF FUNCTIONALIZED SURFACES}

Although the bonding energies of the chemisorbed species are quite large (see Table I), and the presence of the hydrophobic $-\mathrm{CH}_{3}$ group pointing outwards from the surface should inhibit water molecules to reach the surface and promote dissociation of the functional groups from the substrate, we have finally performed a series of finite temperature simulations of water interaction with the functionalized surface. Since most of the relevant technological treatments are performed in wet ambient conditions, this study can provide a rough estimate of the stability of the possible functionalization schemes outlined above, the actual simulation of a functionalized solid/liquid interface being far too complicated and time demanding for the present purposes. Because of the presence of oxygen atoms pointing toward the outer ambient which can enhance interaction with water, we have performed these calculations for chemisorbed acetic acid on $\mathrm{Si}-\mathrm{SiC}(001)$ : we have selected a number of possible sites for an impinging water molecule close to the functional chemisorbed group to maximize the interaction. Our results indicate that water molecules (in the vapor phase) do not react with the functional group, and are either repelled by the $-\mathrm{CH}_{3}$ group, thus flowing away from the surface, or rather dissociate upon the substrate, depending on the starting position, thus supporting our conclusions on the stability of the functionalizations proposed. 


\section{CONCLUSIONS}

In conclusion, we have performed a detailed ab initio study of the adsorption mechanism of selected organic molecules on the silicon terminated stoichiometric $\mathrm{SiC}(001)$ surface, to pave the way towards possible functionalization of this wide-gap semiconductor, with promising characteristics for bio-technology applications. Our results show that at variance with the most exploited semiconductors such as $\mathrm{Si}$ and GaAs, the most common functional groups strongly bind to the surface, as a consequence of the substrate polarity, with exothermal reactions. For example the energy gain estimated for both cleavage mechanisms for methylamine dissociative adsorption on $\mathrm{Si}-\mathrm{SiC}(001)$ are larger than those obtained e.g. for $\mathrm{Si}(001)$ [8]: from this result, we expect a larger stability of the functionalization, for both groups on $\mathrm{SiC}$ rather than Si. Furthermore, the preferential chemisorption of thiolates in particular can lead to the realization of stable self-assembled monolayers, with no requirement of preliminar metallic deposition.

In view of the different reactivity of the studied molecules, we suggest that the $-\mathrm{COOH}$ and -SH groups should be protected in order to prevent unwanted reactions at the surface, when dealing with more complex molecules, containing more than one functional group.

A strategy often used during surface functionalization is the use of linkers at the surface. In the case of the $\mathrm{Si}-\mathrm{SiC}$ surface, when using linkers of the form $\mathrm{NH}_{2}-\mathrm{R}-\mathrm{COOH}$, the -COOH would specifically and spontaneously bind to the surface, leaving the $-\mathrm{NH}_{2}$ as outermost group available for further functionalization. A remarkable example would be the functionalization via aminoacids, which attaching through the - $\mathrm{COOH}$ would always expose their N-terminal part for further processing.

\section{ACKNOWLEDGMENTS}

Use of the computing facilities at CINECA (I) (INFM Parallel Computing Initiative) is gratefully acknowledged. Part of this work was performed under the auspices of the U.S. Department of Energy by University of California Lawrence Livermore National Laboratory under contract No. W-7405-Eng-48. 
* Electronic address: catellani@imem.cnr.it

[1] J. M. Buriak, Chem. Rev. 102, 1271 (2002).

[2] A. Ulman, Chem. Rev 96, 1553 (1996).

[3] G. Poirier, Chem. Rev. 97, 1117 (1997).

[4] F. Schreiber, Prog. Surf. Sci. 65, 151 (2000).

[5] G. Ledung, M. Bergkvust, A. P. Quist, U. Gelius, J. Carlsson, and S. Oscarsson, Langmuir 17, 6056 (2001).

[6] N. Camillone, K. A. Khan, and R. Osgood, Surf. Sci. 453, 83 (2000).

[7] L. Mohaddes-Ardabili, L. J. Martinez-Miranda, J. Silverman, A. Christou, L. G. SalamancaRiba, and M. Al-Sheikhly, Appl. Phys. Lett. 83, 192 (2003).

[8] C. Mui, G. T. Wang, S. Bent, and C. Musgrave, J. Chem. Phys. 114, 10170 (2001).

[9] Y. Shen, L. Guifeng, H. Noda, K. Uosaki, and M. Osawa, Surf. Science 529, 163 (2003).

[10] K. Takahashi, Y. Tomomura, H. Ikeda, and H. Kawanishi, Appl. Phys. Lett. 78, 1364 (2001).

[11] K. T. Park and Y. Gao, Phys. Rev. B 47, 4491 (1993).

[12] G. E. Carter, J. B. Casady, J. Bonds, M. E. Okhuysen, J. D. Scofield, and S. E. Saddow, Mater. Sci. Forum 338-3, 1149 (2000).

[13] F. Amy and Y. J. Chabal, J. Chem. Phys. 119, 6201 (2003).

[14] G. Cicero, A. Catellani, and G. Galli, Phys. Rev. Lett. 93, 016102 (2004).

[15] G. Cicero, A. Catellani, and G. Galli, J. Phys. Chem. 108, 16518 (2004).

[16] J. P. Perdew, K. Burke, and M. Ernzerhof, Phys. Rev. Lett. 77, 3865 (1996).

[17] D. Hamann, Phys. Rev. B 40, 2980 (1989).

[18] R. Car and M. Parrinello, Phys. Rev. Lett. 55, 2471 (1985).

[19] We used the first principles molecular dynamics code GP (F. Gygi, 1998-2003).

[20] A. Catellani and G. Galli, Prog. Surf. Sci. 69, 101 (2002). 
TABLE I: Energy gain (eV/molecule) in the dissociative adsorption process for the reactions described

\begin{tabular}{c|c} 
Molecule & $\mathrm{E}_{\text {gain }}$ \\
\hline $\mathrm{H}_{2} \mathrm{O}$ & 3.5 \\
$\mathrm{CH}_{3}-\mathrm{COOH}$ & 2.7 \\
$\mathrm{CH}_{3}-\mathrm{OH}$ & 3.1 \\
$\mathrm{CH}_{3}-\mathrm{SH}$ & 2.8 \\
$\mathrm{CH}_{3}-\mathrm{NH}_{2}$ via $\mathrm{NH}$ & 2.7 \\
$\mathrm{CH}_{3}-\mathrm{NH}_{2}$ via $\mathrm{CN}$ & 3.3 \\
\hline
\end{tabular}

TABLES 


\section{FIGURE CAPTIONS}

FIG. 1: Ball and stick representation of an interacting water/Si-SiC(001) configuration (side view), before dissociation occurs. Charge polarization at the Si dimer is shown. Only the uppermost substrate layers are shown for clarity; dark (light) gray spheres in the substrate represent silicon (carbon) atoms, while dark (light) gray spheres in the adsorbate represent oxygen (hydrogen) atoms.

FIG. 2: Side view of dissociative adsorption on $\mathrm{Si}-\mathrm{SiC}(001)$ for (right) the acetic acid molecule and (center) methanol. The rightmost panel indicates the adsorption geometry obtained as intermediate physisorbed state. Dark (light) grey spheres in the substrate represent Si (C) atoms, while dark (light) grey spheres in the adsorbate represent $\mathrm{O}(\mathrm{H})$ atoms. Only the outermost planes are shown.

FIG. 3: Side view of the methanethiol molecule chemisorbed at the Si-SiC surface: the metastable (left) and lower energy (right panel) dissociation products are shown. An isosurface of the first state localized on the adsorbed molecule is also shown on the rightmost panel: it resembles the $\mathrm{CH}_{3}$-SH HOMO state. Dark (light) grey spheres in the substrate represent $\mathrm{Si}(\mathrm{C})$ atoms, while dark (light) grey spheres in the adsorbate represent $\mathrm{S}(\mathrm{H})$ atoms. Only the outermost planes are shown. 
FIG. 4: Geometry of the methylamine molecule dissociated at the Si-SiC surface between dimer rows (side view). The left panel represents the adsorption upon CN cleavage, while the right panel figures the NH dissociative adsorption. Dark (light) grey spheres in the substrate represent Si (C) atoms, while dark (light) grey spheres in the adsorbate represent $\mathrm{N}(\mathrm{H})$ atoms. Only the outermost planes are shown. 
FIGURES

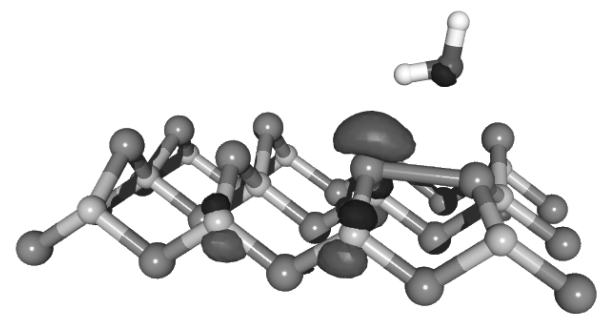

Fig. 1 / Catellani, Cicero 

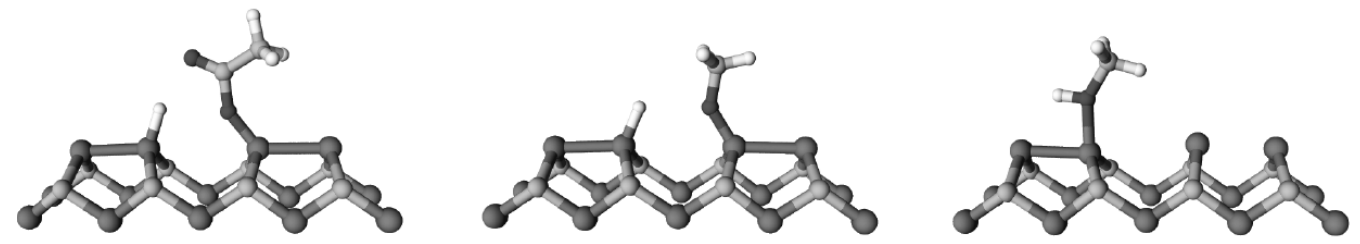

Fig. 2 / Catellani, Cicero 

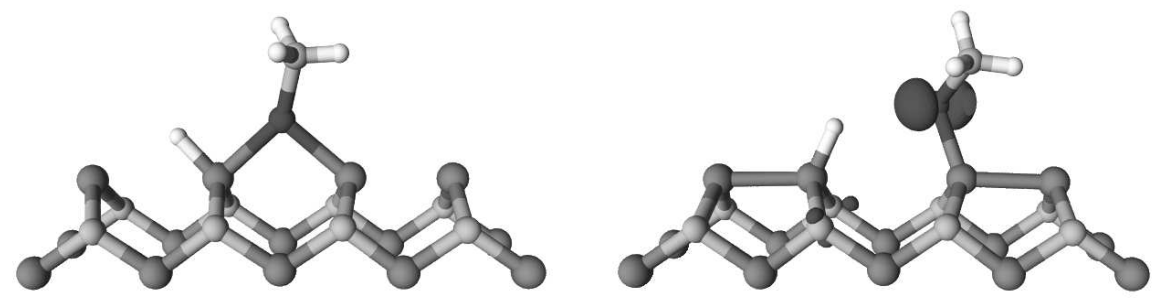

Fig. 3 / Catellani, Cicero 

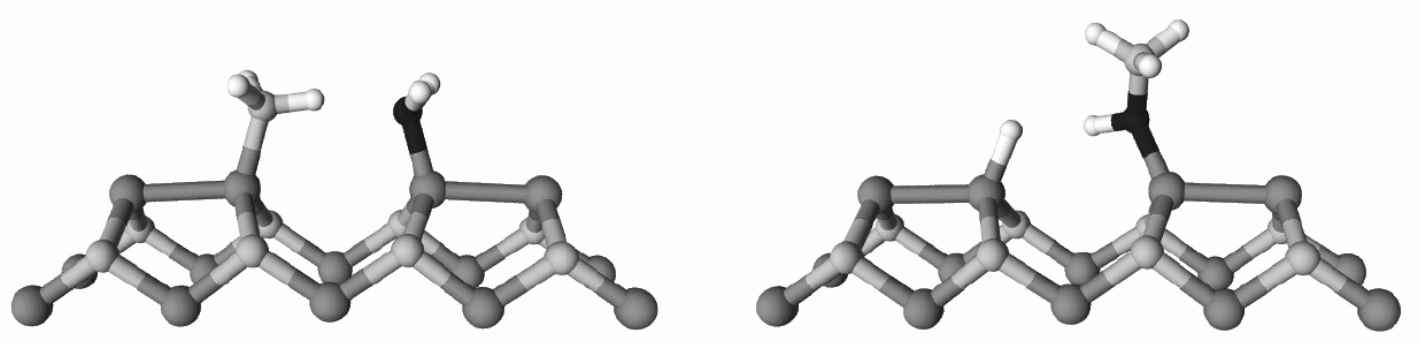

Fig. 4 / Catellani, Cicero 